\title{
Lepton universality, flavour and number violation in $B$ and $\tau$ decays at $\mathrm{LHCb}$
}

\author{
Paula Alvarez Cartelle ${ }^{* \dagger}$ \\ Imperial College London \\ E-mail: paula.alvarez@cern.ch
}

The LHCb experiment has explored lepton universality violation in $b \rightarrow c \ell^{-} \bar{v}_{\ell}$ and $b \rightarrow s \ell^{+} \ell^{-}$ transitions through the measurement of the ratios $R\left(D^{*}\right)$ and $R_{K}$. These ratios are found to be compatible with the Standard Model expectation at the level of $2.1 \sigma$ and $2.6 \sigma$, respectively. Additionally, searches for lepton flavour violation in $B, D$ and $\tau$ decays have been performed. No signal for such decays is found, and upper limits on their branching fractions are set.

9th International Workshop on the CKM Unitarity Triangle

28 November - 3 December 2016

Tata Institute for Fundamental Research (TIFR), Mumbai, India

* Speaker.

${ }^{\dagger}$ on behalf of the LHCb collaboration. 


\section{Introduction}

In the Standard Model (SM), the couplings of the gauge bosons to the leptons are independent of the lepton flavour. This symmetry, so-called lepton flavour universality (LFU), can be however violated in many New Physics (NP) scenarios [1,2]. While lepton flavour violation (LFV) is allowed in the SM with massive neutrinos, the size of lepton-flavour-violating processes induced only by neutrino oscillation is beyond the reach of any current experiment. Consequently, any sign of LFV or lepton flavour non-universality would be a direct sign for physics beyond the SM.

A summary of the latest searches for LFU violation and LFV at LHCb is presented in this document. These measurements are based on the full LHC Run I data sample recorded by the LHCb experiment [3], unless explicitly indicated. The data consists of proton-proton collisions at centre-of-mass energies of 7 and $8 \mathrm{TeV}$ and corresponds to an integrated luminosity of $3.0 \mathrm{fb}^{-1}$.

\section{Lepton flavour universality tests}

The LFU hypothesis has been tested at LHCb by comparing decays of $B$ hadrons into final states that only differ by the lepton content. Semileptonic decays of the type $b \rightarrow c \ell^{-} \bar{v}_{\ell}$ are very abundant and therefore a perfect laboratory for such searches. The observable $R\left(D^{*}\right)$, defined as the ratio of branching fractions of $B^{0} \rightarrow D^{*-} \tau^{+} v_{\tau}$ to $B^{0} \rightarrow D^{*-} \mu^{+} v_{\mu}{ }^{1}$, is predicted to be $0.252 \pm$ 0.003 in the SM [4], and differs from unity mainly because of phase-space effects due to the $\tau-\mu$ mass difference. Previous measurements of this quantity performed by the BaBar and Belle collaborations reported an excess with respect to the SM prediction [5, 6].

At $\mathrm{LHCb}$, the $\tau$ is reconstructed as $\tau^{-} \rightarrow \mu^{-} \bar{v}_{\mu} v_{\tau}$ to minimise differences between both signal decays, and the $D^{*+}$ is reconstructed as $D^{*+} \rightarrow D^{0}\left(K^{-} \pi^{+}\right) \pi^{+}$. A three-dimensional maximum likelihood fit is used to separate the two signal processes from backgrounds due to partially reconstructed $B$ decays, particle misidentification and combinatorial. The three discriminating variables: the missing mass, $m_{\text {miss }}^{2}$, the energy of the muon, $E_{\mu}^{*}$, and the squared four-momentum of the lepton system, $q^{2}$, are evaluated in an approximate rest frame for the $B$ candidate defined from the momentum of the visible decay products. The result of the fit is shown in Fig. 1. The obtained value for $R\left(D^{*}\right)$ is $0.336 \pm 0.027$ (stat) \pm 0.030 (syst), which is consistent with the SM prediction at $2.1 \sigma$.
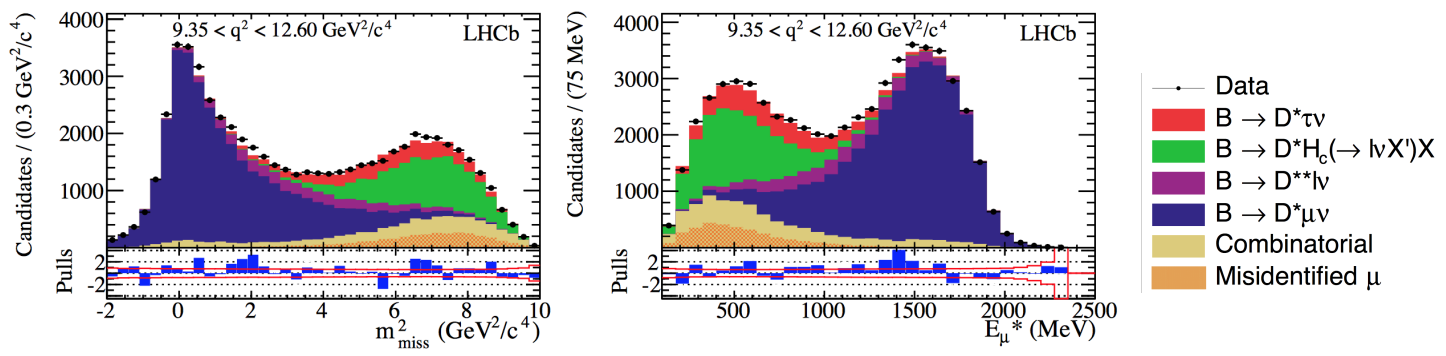

Figure 1: One-dimensional projections of the fit performed to extract the yields of $B^{0} \rightarrow D^{*-} \tau^{+} v_{\tau}$ and $B^{0} \rightarrow D^{*-} \mu^{+} v_{\mu}$. These projections correspond to the highest $q^{2}$ bin only.

\footnotetext{
${ }^{1}$ The inclusion of charge-conjugate processes is implied throughout this document.
} 


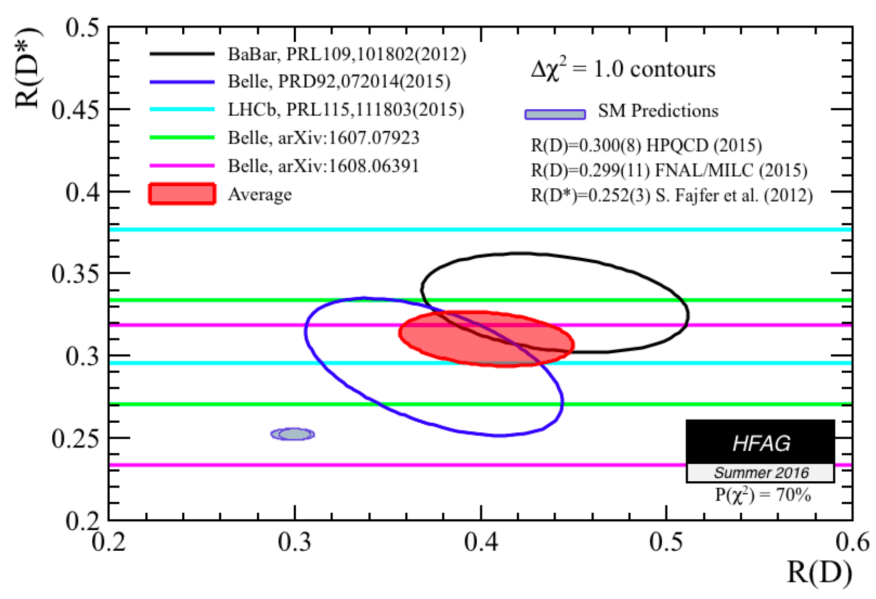

Figure 2: $R(D)$ and $R\left(D^{*}\right)$ measurements and average compared to the SM prediction [7]. The ratio $R(D)$ is equivalent to $R\left(D^{*}\right)$ but computed using $B^{+} \rightarrow D^{0} \ell^{+} v_{\ell}$ decays.

When combined with measurements from other experiments the tension with the SM raises to the $3.9 \sigma$ level [7], as is shown in Fig. 2.

A complementary test for LFU can be performed in the realm of rare decays, such as $b \rightarrow$ $s \ell^{+} \ell^{-}$processes. These flavour changing neutral current transitions are expected to be very sensitive to NP effects as they can only proceed through loop diagrams in the SM. An interesting observable is the ratio of branching fractions between $B^{+} \rightarrow K^{+} \mu^{+} \mu^{-}$and $B^{+} \rightarrow K^{+} e^{+} e^{-}$, hereafter referred to as $R_{K}$, which is predicted to be unity in the $\mathrm{SM}^{2}$ with a precision of $\mathscr{O}\left(10^{-4}\right)$ [8].

LHCb has measured the $R_{K}$ observable in the $q^{2}$ region between 1 and $6 \mathrm{GeV}^{2}$ [11]. The measurement is performed as a double ratio with respect to $B^{+} \rightarrow K^{+} J / \psi\left(\ell^{+} \ell^{-}\right)$in order to reduce systematic uncertainties coming from the different behaviour of muons and electrons in the detector. The yield for the signal and normalisation channels is determined from a maximum likelihood fit to the invariant mass of the three decay products, $m_{K \ell \ell}$. The obtained value is $R_{K}=0.745_{-0.074}^{+0.090}$ (stat) \pm 0.036 (syst), and is compatible with the SM expectation at the level of $2.6 \sigma$, see Fig. 3. This small tension, although not yet significant, has been claimed to be consistent with other anomalies observed in the measurement of branching fractions and angular observables in other $b \rightarrow s \ell^{+} \ell^{-}$transitions, such as $B^{0} \rightarrow K^{* 0} \mu^{+} \mu^{-}$[14] or $B_{s}^{0} \rightarrow \phi \mu^{+} \mu^{-}$[15]. Global fits to all of these observables would favour a shift of the Wilson coefficient $C_{9}$ with respect to the SM expectation, with a significance of around $4 \sigma[16,17,18]$. Moreover, unlike other $b \rightarrow s \ell^{+} \ell^{-}$ observables, $R_{K}$ is essentially free from hadronic uncertainties. Future updates of this quantity and measurements of related LFU ratios are therefore crucial to clarify whether NP is needed to explain these anomalies.

Further tests of LFU in rare decays come from the study of $B_{(s)}^{0} \rightarrow \ell \ell$ decays. After the first observation of the $B_{s}^{0} \rightarrow \mu \mu$ decay by the LHCb and CMS collaborations [19], a comparison of its branching fraction with that of the $B_{s}^{0} \rightarrow \tau \tau$ decay would allow to test lepton universality. LHCb has performed a search for the decays $B_{s}^{0} \rightarrow \tau \tau$ and $B^{0} \rightarrow \tau \tau$, where the $\tau$ is reconstructed through its

\footnotetext{
${ }^{2}$ Electromagnetic corrections have been estimated to be of the order of 3\% [9] and are taken into account in this analysis using PHOTOS [10].
} 


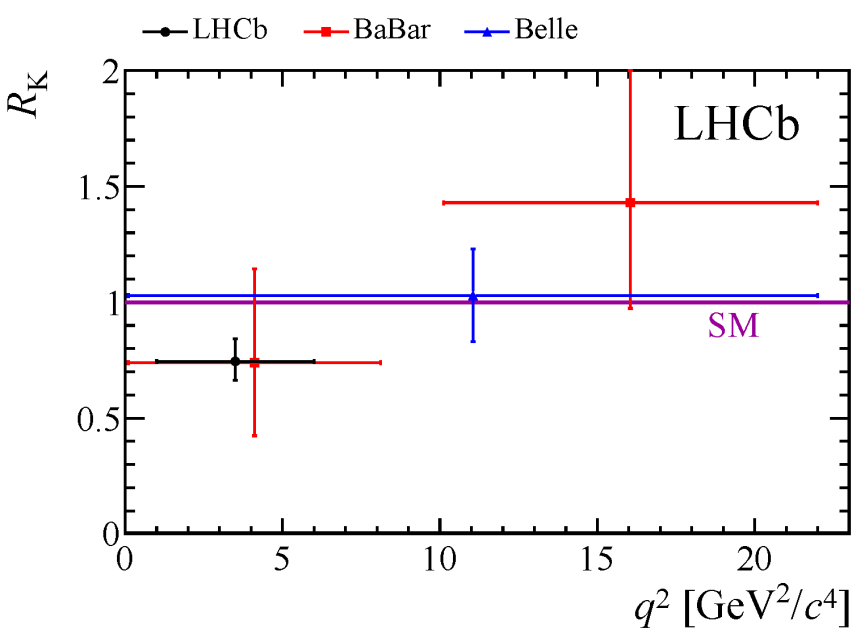

Figure 3: Measurements of $R_{K}$ performed by LHCb [11], BaBar [12] and Belle [13].

hadronic decay into three charged pions and a neutrino, assuming specific intermediate resonances: $\tau^{-} \rightarrow a_{1}^{-}\left(\rho^{0} \pi^{-}\right) v_{\tau}$. A multivariate classifier combines kinematic and topological information of the decay and allows the signal yield to be extracted. No signal is observed, and the 95\% C.L. upper limits $B\left(B_{s}^{0} \rightarrow \tau \tau\right)<3.0 \times 10^{-3}$ and $B\left(B^{0} \rightarrow \tau \tau\right)<1.3 \times 10^{-3}$ are set [20].

\section{Lepton flavour violation searches}

A number of NP scenarios predict LFV decays at branching fractions approaching current experimental sensitivities [21]. If charged LFV were to be discovered, measurements of the branching fractions for a number of channels would be required to determine the nature of the NP model. In the absence of such a discovery, improving the experimental constraints on the branching fractions of LFV decays would help to constrain the parameter space of these models.

The branching fraction of the decay $D^{0} \rightarrow e^{ \pm} \mu^{ \pm}$is predicted to be enhanced in many NP models, from R-parity violating supersymmetric models to extensions of the SM with multiple Higgs doublets [22], reaching values up to $\mathscr{O}\left(10^{-6}\right)$. LHCb has performed a search for this decay, selecting candidates produced via the $D^{*+} \rightarrow D^{0} \pi^{+}$decay chain, and using as normalisation the well-measured decay channel $D^{0} \rightarrow K^{-} \pi^{+}$, which has the same topology as the signal. A multivariate analysis based on a boosted decision tree algorithm (BDT) is used to help separate signal and background. A two dimensional fit in the mass of the $e \mu$ pair and the mass difference between the $D^{* 0}$ and the $D^{0}$ candidates is performed in bins of the BDT, and no signal is found. Therefore, the upper limits $B\left(D^{0} \rightarrow e^{ \pm} \mu^{ \pm}\right)<1.3(1.6) \times 10^{-8}$ at $90(95) \%$ C.L. are set [23], see Fig. 4. This limit improves the previous best limit from Belle [24] by an order of magnitude.

In addition, $\mathrm{LHCb}$ previously set limits on branching fractions for the $B$ meson decays $B_{(s)}^{0} \rightarrow$ $e^{ \pm} \mu^{ \pm}$[25]. These decays are allowed in several scenarios beyond the SM, but, in particular, they could be mediated via exchange of spin-1 gauge bosons, the so-called Pati-Salam leptoquarks, that carry both colour and lepton quantum numbers [26]. The limits obtained using $1.0 \mathrm{fb}^{-1}$ of LHCb data, $B\left(B_{(s)}^{0} \rightarrow e^{ \pm} \mu^{ \pm}\right)<2.8(1.1) \times 10^{-9}$ at $90 \%$ C.L., are used to derive lower limits on the masses of these leptoquarks [25]. 

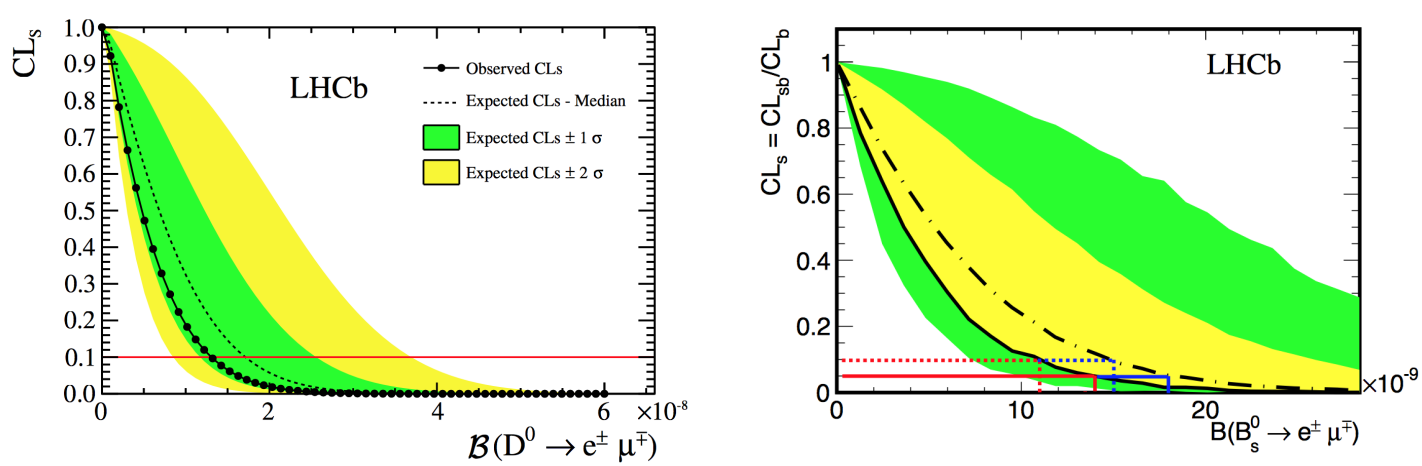

Figure 4: Expected (dashed line) and observed (solid line) CLs distributions as a function of the branching fraction of $D^{0} \rightarrow e^{ \pm} \mu^{ \pm}$(left) and $B_{(s)}^{0} \rightarrow e^{ \pm} \mu^{ \pm}$(right) decays. The horizontal lines indicate the $90 \%$ and 95\% confidence levels in each case.

In the lepton sector, the decay $\tau^{-} \rightarrow \mu^{-} \mu^{+} \mu^{-}$is particularly interesting for NP models involving an extended Higgs sector [27], since they feature enhanced lepton flavour violating couplings to heavy leptons. LHCb has presented a search for the $\tau^{-} \rightarrow \mu^{-} \mu^{+} \mu^{-}$decay using as normalisation channel the decay $D_{s} \rightarrow \phi \pi^{-}$with $\phi \rightarrow \mu^{+} \mu^{-}$. Discrimination between a potential signal and the background is performed using a three-dimensional binned distribution in two multivariate classifiers and the invariant mass of the $\tau$ candidate. The first classifier is based on the three-body decay topology. The second exploits muon identification information. No evidence for an excess over the background expectation is found and only an upper limit is established. The measured limit, $B\left(\tau^{-} \rightarrow \mu^{-} \mu^{+} \mu^{-}\right)<4.6 \times 10^{-8}$ at $90 \%$ C.L. [28], supersedes the previous measurement from LHCb [29] and, in combination with results from the $B$ factories [7], improves the constraints placed on the parameters of a broad class of NP models.

\section{Conclusions}

The observation of lepton flavour non-universality or lepton flavour violation would be a clear signature of New Physics. Two LHCb tests of lepton universality show hints of deviations from the SM expectation. The $R\left(D^{*}\right)$ measurement is in agreement with what is found by BaBar and Belle and, combined with those, it exceeds the SM prediction by 3.9 $\sigma$. In the rare decays sector, $R_{K}$ shows also a small discrepancy with unity; this coherently fits the pattern of other LHCb measurements in $b \rightarrow s \ell^{+} \ell^{-}$transitions, pointing to possible NP involving the Wilson coefficient $C_{9}$. The improvement of these measurements and the study of additional $b \rightarrow c \ell^{-} \bar{v}_{\ell}$ and $b \rightarrow s \ell^{+} \ell^{-}$ processes are, therefore, of great interest. So far, no lepton flavour violation has been observed in $B, D$ or $\tau$ decays, but upper limits on the branching fractions of $D^{0} \rightarrow e^{ \pm} \mu^{ \pm}, B_{(s)}^{0} \rightarrow e^{ \pm} \mu^{ \pm}$and $\tau^{-} \rightarrow \mu^{-} \mu^{+} \mu^{-}$decays have been provided by LHCb. Further constraining these limits will help to narrow the allowed parameter space of multiple NP models.

\section{References}

[1] G. Hiller and M. Schmaltz, Phys. Rev. D90 (2014) 054014, arXiv:1408.1627

[2] M. Tanaka, Z. Phys. C67 (1995) 321, arXiv:hep-ph/9411405 
[3] LHCb collaboration, JINST 3 (2008) S080005,

[4] S. Fajfer et al, Phys. Rev. D85 (2012) 094025

[5] BaBar Collaboration, Phys. Rev. D88 (2013) 072012, arXiv:1303.0571

[6] Belle Collaboration, Phys. Rev. D 94 (2016) 072007, arXiv:1607.07923

[7] Heavy Flavor Averaging Group, arXiv:1612.07233

[8] C. Bobeth et al, JHEP 12 (2007), arXiv:0709.4174

[9] M. Bordone et al, A. Eur. Phys. J. C76 (2016) 440, arXiv:1605.07633

[10] P. Golonka and Z. Was, Eur. Phys. J. C45 (2006) 97, arXiv:hep-ph/0506026

[11] LHCb collaboration, Phys. Rev. Lett. 113 (2014) 151601, arXiv:1406.6482

[12] BaBar Collaboration, Phys. Rev. D86 (2012) 032012, arXiv:1204.3933

[13] Belle collaboration, Phys. Rev. D103 (2009) 12, arXiv:0810.0335

[14] LHCb Collaboration, JHEP 02 (2016) 104, arXiv:1512.04442

[15] LHCb collaboration, JHEP 09 (2015) 179, arXiv:1506.08777

[16] S. Descotes-Genon et al, JHEP 06 (2016) 092, arXiv:1510.04239

[17] T. Hurth et al, JHEP 1412 (2014) 053, arXiv:1410.4545

[18] W. Altmannshofer and D. M. Straub, Eur. Phys. J. C 75 (2015) 382, arXiv:1411.3161

[19] CMS and LHCb collaborations, Nature 522 (2015) 68, arXiv:1411.4413

[20] LHCb collaboration, LHCb-CONF-2016-011

[21] W. J. Marciano et al, Ann. Rev. Nucl. Part. Sci 58 (2008) 315

[22] G. Burdman et al, Phys. Rev. D66 (2002) 014009, arXiv:hep-ph/0112235.

[23] LHCb collaboration, Phys. Lett. B754 (2016) 167, arXiv:1512.00322

[24] Belle collaboration, Phys. Rev. D81, (2010) 091102, arXiv:1003.2345

[25] LHCb collaboration, Phys. Rev. Lett. 111 (2013) 141801, arXiv:1307.4889

[26] J. C. Pati and A. Salam, Phys. Rev. D10 (1974) 275

[27] M. Blanke et al, Acta Phys.Polon. B41 (2010) 657, arXiv:0906.5454

[28] LHCb collaboration, JHEP 02 (2015) 121, arXiv:1409.8548

[29] LHCb collaboration, Phys. Lett. B724 (2013) 36, arXiv:1304.4518. 